\title{
Advocacia em saúde na atenção à criança: revisão da literatura
}

\author{
Health advocacy in child care: literature review \\ Abogacía en salud en la atención al niño: revisión de la literatura
}

\section{Raquel Dully Andrade', Débora Falleiros Mello", Marta Angélica lossi Silva", Carla Aparecida Arena Ventura'II}

\author{
' Universidade de São Paulo, Escola de Enfermagem de Ribeirão Preto-USP, \\ Programa Pós-Graduação em Enfermagem em Saúde Pública (Doutoranda). Ribeirão Preto-SP, Brasil. \\ "Universidade de São Paulo, Escola de Enfermagem de Ribeirão Preto-USP, \\ Departamento Enfermagem Materno Infantil e Saúde Pública. Ribeirão Preto-SP, Brasil. \\ I'I Universidade de São Paulo, Escola de Enfermagem de Ribeirão Preto-USP, \\ Departamento de Enfermagem Psiquiátrica e Ciências Humanas. Ribeirão Preto-SP, Brasil.
}

Submissão: 23/12/2009 Aprovação: 29/05/2010

\section{RESUMO}

Revisão narrativa da literatura, a partir das bases de dados LILACS e MEDLINE, entre 2004 e 2009, com a qual se objetivou identificar as publicações relativas à temática da advocacia em saúde no âmbito da atenção à saúde da criança. Foram analisados treze artigos e identificados três temas: Ênfase em conhecimentos, habilidades e atitudes para desenvolvimento de competências; Parcerias como um imperativo; Saúde e Direito: relação intersetorial. Os estudos sobre o exercício da advocacia em saúde são relevantes para nossa realidade, especialmente na atenção primária à saúde, chamando a atenção para as possibilidades de sua aplicabilidade no papel do enfermeiro atuante na estratégia saúde da família, junto a famílias e crianças.

Descritores: Advocacia em saúde; Criança; Família; Enfermagem.

ABSTRACT
This narrative literature review aimed to identify the publications about health law, in the ambit of child health care. The databases LILACS and MEDLINE were searched, between 2004 and 2009. Thirteen articles were analyzed, and three themes were identified: Emphasis on knowledge, abilities and attitudes for the development of competencies; Partnerships as an imperative; Health and Law: intersectorial relationship. The studies about the practice of health law are relevant to our reality, especially in primary health care, pointing out for the possibilities of its applicability in the role of the nurses acting in the family health strategy, with families and children.

Key words: Health law; Children; Family; Nursing.

\section{RESUMEN}

Revisión narrativa de la literatura, por búsquedas en las bases de datos LILACS y MEDLINE, entre 2004 y 2009 , con que se intentó identificar las publicaciones relativas a la temática de la abogacía en salud en el ámbito de la atención a la salud del niño. Fueron analizados trece artículos e identificados tres temas: Énfasis en conocimientos, habilidades y actitudes para desarrollo de competencias; Parecerías como un imperativo; Salud y Derecho: relación intersectorial. Los estudios sobre el ejercicio de la abogacía en salud son relevantes para nuestra realidad, especialmente en la atención primaria a la salud, Ilamando la atención para las posibilidades de su aplicabilidad en el papel del enfermero actuante en la estrategia de salud familiar, junto a familias y niños. Palabras clave: Defensa de la salud; Niño; Familia; Enfermería. 


\section{INTRODUÇÃO}

Atualmente, na área de conhecimento da saúde, considera-se que o indivíduo, antes objeto de intervenção e prática eminentemente clínica, deve ser compreendido como sujeito incluído em contextos sócio, econômico, físico, político e cultural. Desse modo, cabe ao enfermeiro, atuar na promoção e proteção da saúde e do ser humano como um todo, respeitando os preceitos éticos e legais, para a observância e garantia dos direitos de cidadania ${ }^{(1)}$. Em acordo com esse entendimento está o conceito de advocacia em saúde.

As primeiras experiências de advocacia em saúde são demarcadas por um movimento de reivindicação de um grupo de pediatras para a garantia dos direitos das crianças ${ }^{(2)}$. Assim, observa-se que a utilização do termo advocacia na área da saúde nasceu em um campo de atenção à clientela infantil e que foi por meio de profissionais de saúde que as primeiras experiências aconteceram.

Relacionando advocacia e situações de vulnerabilidade, destaca-se o grupo etário infantil. Um estudo ${ }^{(3)}$, discorrendo sobre a atuação profissional em pediatria, afirma que essa área vê a criança, a família e a comunidade como um contínuo e menciona o papel de "conselheiro e advogado", enfatizando o "advogar pela causa" como uma arte e uma ciência.

Existem instrumentos legais para que os direitos humanos sejam assegurados. No Brasil, segundo a Constituição Federal de 1988, a saúde é um direito de todos e um dever do Estado. Inclui também artigos que prevêem formas de defesa, os quais o cidadão pode lançar mão nos casos em que os direitos são violados $^{(4)}$. Entretanto, tais direitos são pouco conhecidos tanto pelos usuários quanto pelos profissionais de saúde, levando a um contexto em que, mesmo que exista uma legislação adequada, essa não seja aplicada na prática.

O Estatuto da Criança e do Adolescente (ECA), promulgado nos anos 90 do século $\mathrm{XX}$, é uma das maiores conquistas da sociedade civil organizada. O ECA segue a doutrina da proteção integral da criança e do adolescente, propagada pela Organização das Nações Unidas. No entanto, colocar seus propósitos em prática é tomado como um grande desafio ${ }^{(5)}$.

O termo advocacia em saúde pode estar associado à consolidação de outros conceitos da área da saúde, como o de promoção da saúde, intersetorialidade, gestão da clínica, sistemas integrados de saúde, controle social, participação popular, entre outros, com contribuições para ampliar a saúde das crianças e de suas famílias.

Este estudo tem por objetivo identificar, em periódicos científicos nacionais e internacionais, as publicações relativas à temática da advocacia em saúde no âmbito da atenção à saúde da criança.

\section{METODOLOGIA}

Esta investigação configura-se como um estudo bibliográfico, com base em uma revisão narrativa da literatura. A revisão narrativa da literatura busca reunir dados sobre uma temática de forma mais aberta ${ }^{(6)}$.
A questão norteadora adotada para este estudo foi: qual é o conhecimento científico produzido acerca da temática de advocacia em saúde com enfoque na atenção à saúde da criança? A busca dos artigos foi feita nas bases de dados LILACS e MEDLINE, tendo como limite de publicação o período 2004 a 2009. No LILACS, foram utilizadas as palavras chave: "advocacia em saúde", "direito à saúde" e "defesa do paciente", e seus respectivos sinônimos em espanhol, que constituem descritores cadastrados no DeCS (Descritores em Ciências da Saúde); no MEDLINE, foram utilizadas as palavras-chave "health advocacy" juntamente com "child".

A inclusão dos trabalhos foi baseada nos seguintes critérios: resumo disponível nas bases de dados acima descritas; idioma de publicação em português, inglês ou espanhol; publicação no período proposto. Foram excluídos os estudos que apenas citavam o termo advocacia em saúde e que não discutiam o tema, e os que tratavam do conceito de forma direcionada a situações, condições ou morbidades específicas, pois estes poderiam se diferenciar nos resultados dos trabalhos que abordavam esse conceito de forma mais generalizada. No LILACS, foram encontrados dezessete (17) artigos sobre advocacia em saúde, sendo doze (12) em português e cinco (5) em inglês, mas nenhum deles eram relacionados à criança e, por isso, não foram incluídos nesse estudo. No MEDLINE, foram encontrados 96 trabalhos, e a partir da leitura dos resumos, foram selecionados treze (13) artigos que atendiam aos critérios de inclusão para a presente revisão narrativa.

A partir dessa seleção foi realizada a localização dos textos na íntegra. Após a análise deles, foi realizada uma síntese dos dados, contemplando ano de publicação, título do periódico, autores, país, objetivo, metodologia, principais resultados e conclusões.

\section{RESULTADOS}

Os treze (13) artigos selecionados estavam escritos no idioma inglês e alguns dados que caracterizam o perfil dessas produções estão apresentados no Quadro a seguir.

Em relação aos periódicos que se constituem como fonte dos artigos selecionados, observa-se uma concentração naqueles da área de Pediatria (nove artigos), seguido de três artigos da área da Medicina, de uma forma geral, e um periódico da área de Enfermagem.

Em relação ao país, destacou-se os Estados Unidos da América, com sete artigos, seguido da Inglaterra com quatro, e Canadá e Austrália com um trabalho cada.

Em relação aos objetivos dos artigos selecionados para esta revisão, observou-se uma concentração em temáticas voltadas ao ensino de Pediatria Comunitária em programas de residência médica; estando presentes também os temas de atenção primária à saúde e serviços de advocacia; atuação de enfermagem a famílias no âmbito da atenção primária à saúde; atenção à criança através de visitas domiciliares em saúde; saúde da criança e direitos humanos. Observa-se que há uma expressiva ligação dos objetos de estudo ao campo da atenção primária à saúde.

No tocante aos métodos empregados no desenvolvimento dessas investigações, há abordagens quantitativa e qualitativa, 


\begin{tabular}{|c|c|c|c|c|c|c|}
\hline No & Ano & Periódico Fonte & Autor(es) & País & Objetivo & Método \\
\hline 1 & 2009 & Acad Med. & $\begin{array}{l}\text { Hufford L, West DC, } \\
\text { Paterniti DA, Pan RJ. }\end{array}$ & $\begin{array}{l}\text { Estados } \\
\text { Unidos }\end{array}$ & $\begin{array}{l}\text { Descrever o currículo de uma nova abordagem, } \\
\text { para ensinar médicos residentes em pediatria } \\
\text { a serem advogados efetivos de comunidades, } \\
\text { chamada de "Comunidades e Clínicos Juntos" } \\
\text { (Community and Physicans Together- CPT }{ }^{(7)}\end{array}$ & $\begin{array}{l}\text { Descritivo- } \\
\text { qualitativo }\end{array}$ \\
\hline 2 & 2009 & $\begin{array}{l}\text { Arch Dis Child } \\
\text { Educ Pract Ed. }\end{array}$ & Waterston $\mathrm{T}$. & Inglaterra & $\begin{array}{l}\text { Sugerir meios para incluir treinamento de advocacia } \\
\text { no núcleo de ensino de pediatras, através da } \\
\text { combinação de ensino teórico e experiência prática }\end{array}$ & $\begin{array}{l}\text { Relato de } \\
\text { Experiência }\end{array}$ \\
\hline 3 & 2007 & $\begin{array}{l}\text { Paediatr Child } \\
\text { Health. }\end{array}$ & $\begin{array}{l}\text { Au H, Harrison M, } \\
\text { Ahmet A, Orsino A, } \\
\text { Beck CE, Tallet S, } \\
\text { Gans M, Birken CS. }\end{array}$ & Canadá & $\begin{array}{l}\text { Descrever o desenvolvimento, a implementação e } \\
\text { avaliação dos primeiros quatro anos da iniciativa } \\
\text { de advocacia da criança da Universidade de } \\
\text { Toronto }^{(9)}\end{array}$ & $\begin{array}{l}\text { Revisão de } \\
\text { Literatura, Grupos } \\
\text { Focais e Pesquisa } \\
\text { Quantitativa }\end{array}$ \\
\hline 4 & 2008 & Ambul Pediatr. & $\begin{array}{l}\text { Keller D, Jones N, } \\
\text { Savageau JA, Cashman SB. }\end{array}$ & $\begin{array}{l}\text { Estados } \\
\text { Unidos }\end{array}$ & $\begin{array}{l}\text { Determinar se um questionário de triagem para } \\
\text { advocacia médico-jurídica (MASQ) é capaz de } \\
\text { identificar famílias, no âmbito da atenção primária } \\
\text { à saúde, com necessidades de referenciamento } \\
\text { a serviços de advocacia que possam melhorar a } \\
\text { saúde da criança de forma mais efetiva do que a } \\
\text { intervenção clínica isolada }{ }^{(10)}\end{array}$ & Quantitativo \\
\hline 5 & 2007 & Med Teach. & $\begin{array}{l}\text { Grant N, Gibbs T, } \\
\text { Naseeb TA, Al-Garf A. }\end{array}$ & Inglaterra & $\begin{array}{l}\text { Avaliar o desenvolvimento de um programa para } \\
\text { estudantes médicos desenvolverem habilidades } \\
\text { e competências como advogados de saúde da } \\
\text { família }^{(11)}\end{array}$ & Quantitativo \\
\hline 6 & 2007 & $\begin{array}{l}\text { J Paediatr Child } \\
\text { Health. }\end{array}$ & $\begin{array}{l}\text { Raman S, Woolfenden S, } \\
\text { Williams K, Zwi K. }\end{array}$ & Austrália & $\begin{array}{l}\text { Discutir a proteção do bem-estar e da saúde da } \\
\text { criança sob a ótica dos Direitos Humanos }{ }^{(12)}\end{array}$ & ReflexãoTeórica \\
\hline 7 & 2007 & Ambul Pediatr. & $\begin{array}{l}\text { Solomon BS, Minkovitz } \\
\text { CS, Grason HA, } \\
\text { Carraccio C. }\end{array}$ & $\begin{array}{l}\text { Estados } \\
\text { Unidos }\end{array}$ & $\begin{array}{l}\text { Avaliar os Programas de Residência Pediátrica em } \\
\text { relação ao treinamento de pediatria comunitária } \\
\text { no período de } 2002 \text { a } 2005^{(13)}\end{array}$ & Quantitativo \\
\hline 8 & 2007 & J Adv Nurs. & Cohen BE, Reutter L. & Inglaterra & $\begin{array}{l}\text { Discutir o papel de enfermeiros da atenção } \\
\text { primária à saúde junto a famílias e crianças } \\
\text { vivendo em situação de pobreza }{ }^{(14)}\end{array}$ & $\begin{array}{c}\text { Revisão de } \\
\text { Literatura e } \\
\text { Reflexão Teórica }\end{array}$ \\
\hline 9 & 2007 & $\begin{array}{l}\text { Health Soc Care } \\
\text { Community. }\end{array}$ & McIntosh J, Shute J. & Inglaterra & $\begin{array}{l}\text { Avaliar as visitas domiciliares em saúde e seus } \\
\text { resultados nas percepções de pais a respeito de } \\
\text { receber apoio dos profissionais de saúde }\end{array}$ & Qualitativo \\
\hline 10 & 2006 & Ambul Pediatr. & $\begin{array}{l}\text { MinKovitz CS, Chandra A, } \\
\text { Solomon BS, McDonnell } \\
\text { KA, Silver GB, } \\
\text { Tonniges TF. }\end{array}$ & $\begin{array}{l}\text { Estados } \\
\text { Unidos }\end{array}$ & $\begin{array}{l}\text { Avaliar as diferenças de gênero no envolvimento } \\
\text { de médicos residentes pediatras nas atividades } \\
\text { comunitárias }^{(16)}\end{array}$ & Quantitativo \\
\hline 11 & 2006 & Acad Méd. & $\begin{array}{l}\text { Paterniti DA, Pan RJ, } \\
\text { Smith LF, Horan NM, } \\
\text { West DC. }\end{array}$ & $\begin{array}{l}\text { Estados } \\
\text { Unidos }\end{array}$ & $\begin{array}{l}\text { Compreender a influencia do bloco de ensino da } \\
\text { advocacia da criança na comunidade, sobre as } \\
\text { perspectivas a respeito da advocacia em saúde, } \\
\text { nos primeiros } 5 \text { anos enquanto residentes em } \\
\text { pediatria e se essa influência persiste após o } \\
\text { término desse período de formação } o^{(17)}\end{array}$ & Qualitativo \\
\hline 12 & 2005 & $\begin{array}{l}\text { Arch Pediatr } \\
\text { Adolesc Med. }\end{array}$ & $\begin{array}{l}\text { Chamberlain LJ, } \\
\text { Sanders LM, Takayama JI. }\end{array}$ & $\begin{array}{l}\text { Estados } \\
\text { Unidos }\end{array}$ & $\begin{array}{l}\text { Descrever escolhas dos residentes em pediatria a } \\
\text { respeito de tópicos e intervenções de advocacia }{ }^{(18)}\end{array}$ & $\begin{array}{l}\text { Descritivo- } \\
\text { quantitativo }\end{array}$ \\
\hline 13 & 2005 & Pediatrics. & $\begin{array}{l}\text { Shipley LJ, Stelzner SM, } \\
\text { Zenni E A, Hargunani } \\
\text { D, O' Keefe J, Miller C, } \\
\text { Alversom B, Swigonski N. }\end{array}$ & $\begin{array}{l}\text { Estados } \\
\text { Unidos }\end{array}$ & $\begin{array}{l}\text { Descrever abordagens para promover o ensino } \\
\text { sobre saúde comunitária e advocacia da criança }{ }^{(19)}\end{array}$ & Descritivo \\
\hline
\end{tabular}

Quadro 1 - Distribuição das referências incluídas na revisão, conforme ano de publicação, título do periódico, autores, país de origem, objetivos e método utilizado. 
incluindo estudos descritivos, reflexão teórica, revisão bibliográfica e relato de experiência.

A análise das publicações na íntegra permitiu a identificação de três unidades temáticas presentes nas discussões sobre advocacia em saúde da criança, sendo: Ênfase em conhecimentos, habilidades e atitudes para desenvolvimento de competências; Parcerias como um imperativo; Saúde e Direito: relação intersetorial.

Ênfase em conhecimentos, habilidades e atitudes no desenvolvimento de competências

A iniciativa de advocacia da criança deve ser incorporada no currículo de residência para ajudar futuros pediatras a desenvolver competências em advocacia. Através dela, residentes em pediatria tiveram a oportunidade de desenvolver habilidades em advocacia e aprender sobre determinantes de saúde da criança ${ }^{(9,19)}$.

Pedagogicamente, a advocacia é melhor ensinada usando uma abordagem baseada em competências, em que a chave do aprendizado é a definição de habilidades e atitudes, sendo que para isso, é desejável que o aprendizado ocorra através de experiência prática ${ }^{(8,19)}$.

O papel de advogado da saúde da família é uma competência a ser desenvolvida, componente esse importante em um currículo baseado em problemas, de um programa de saúde da comunidade, composto de estudos familiares para a saúde materno-infantil, no qual a ciência está interligada a habilidades profissionais ${ }^{(11)}$. Na avaliação de projetos desenvolvidos pelos residentes, identificou-se a criatividade como uma das competências para adaptar soluções de advocacia necessárias às complexas questões de saúde da criança ${ }^{(18)}$.

Atitudes quanto a seu papel como pediatra na comunidade mudou com o exercício da advocacia em saúde ${ }^{(7)}$. A concepção de residentes sobre advocacia em saúde da criança, após participarem do programa de treinamento, concentrou-se na mudança de idéias sobre ser um pediatra na comunidade (antes) para ser um pediatra para a comunidade (depois) ${ }^{(17)}$.

Um estudo sobre o papel de enfermeiros de saúde pública junto a famílias e crianças em situação de pobreza enfatiza o exercício da advocacia político-cidadã por esse profissional, entretanto, destaca que na revisão de literatura realizada, esse papel foi pouco identificado em padrões e competências profissionais do enfermeiro e, também, poucos trabalhos foram encontrados com evidências empíricas nesse sentido, sugerindo a necessidade de pesquisas que documentem esforços de enfermeiros de saúde pública no engajamento de atividades de advocacia política-cidadã em prol de famílias de crianças vivendo em situação de pobreza. Foram associados ao envolvimento limitado do enfermeiro em atividades de advocacia, a estrutura conceitual e suporte organizacional insipientes, dificultando o desenvolvimento de conhecimentos e habilidades ${ }^{(14)}$.

\section{Parcerias como um imperativo}

Por meio de programas específicos na formação e capacitação de profissionais de saúde há um processo de aprendizagem para tornarem-se parceiros da comunidade ${ }^{(9,17,19)}$.
A advocacia em pediatria comunitária destaca o envolvimento com escolas, centros de cuidado à criança e grupos de proteção infantil ${ }^{(13)}$. A ênfase no estabelecimento de parcerias com membros da comunidade e organizações de proteção infantil são ressaltados para melhorar a saúde da criança ${ }^{(7,17,19)}$. A identificação de escolas para parcerias apropriadas no desenvolvimento de projetos de advocacia da criança, bem como grupos de pais e de professores, são estimulados na identificação de áreas nas quais os profissionais de saúde devem desenvolver envolvimento ${ }^{(9)}$.

Prestação de serviços em saúde que enfatizam a identificação e utilização de forças e potencialidades da comunidade, assim como a capacidade de desenvolvimento e incrementação de estratégias para a resolução de problemas ${ }^{(7)}$. Profissionais identificam recursos da comunidade e desempenham atividades designadas para melhorá-los ${ }^{(7,19)}$, com uma perspectiva de membro da comunidade ${ }^{(7)}$. Membros da comunidade funcionam como facilitadores primários para essa identificação $0^{(7,17,19)}$.

No exercício da advocacia da família, mostrou-se valiosa a capacidade de oferecimento de ajuda prática sobre o uso de recursos da comunidade ${ }^{(11,17)}$. O enfermeiro de saúde pública, no exercício da advocacia política, assistindo famílias vivendo em pobreza, deve identificar serviços apropriados que dirijam esforços no nível político, devendo desempenhar estratégias articuladas dentro da estrutura na qual atua ${ }^{(14)}$.

Redução do isolamento é destacada a partir da visita domiciliar em saúde e, a partir dela, a busca de recursos e serviços para os problemas vivenciados pela família, advogando-se por ela e por suas crianças ${ }^{(15)}$.

\section{Saúde e Direito: relação intersetorial}

A advocacia da criança pelo profissional de saúde envolve tanto questões de saúde quanto de doença, sendo comum a necessidade de articulação com agências de advocacia para abordar as questões que se apresentam diante dos profissionais de saúde ${ }^{(8)}$.

Faz parte do conteúdo ministrado dentro da proposta de ensino-aprendizagem para residentes em pediatria o documento da Convenção dos Direitos da Criança (Nações Unidas), introduzindo assim temas não específicos da área médica ou do setor saúde ${ }^{(8)}$.

Na advocacia para melhorar a saúde e o bem-estar da criança, a abordagem dos Direitos Humanos e o uso da Convenção dos Direitos das Crianças, o qual caracteriza um tratado das Nações Unidas, oferecem uma estrutura originária da área do Direito, adicional para a atuação no setor saúde. Dessa forma, é importante que profissionais de saúde tenham conhecimentos sobre esses instrumentos legais para que possam divulgá-los e utiliza-los na defesa das crianças que assistem ${ }^{(12)}$.

Um instrumento de triagem, como um questionário, pode auxiliar o profissional de saúde a identificar famílias com necessidades de referenciamento para serviços de advocacia, tornando a assistência mais efetiva do que a intervenção clínica isoladamente. O questionário é utilizado na rotina de visita domiciliar de cuidado à saúde da criança e esse instrumento é respondido pelos pais ou cuidadores das crianças assistidas. 
Uma pesquisa, na qual 255 pais responderam o questionário, 85 famílias foram encaminhadas a serviços de advocacia, enquanto apenas 35 dessas foram identificadas quanto a essa necessidade de referenciamento antes da aplicação do instrumento, apenas pela percepção do profissional em seu cuidado primário de saúde. O Centro de Advogados de Famílias de Massachussets é uma colaboração entre médicos e advogados que assistem famílias de baixa renda nos assuntos que afetam a saúde da criança ${ }^{(10)}$.

A identificação da inclusão de ensino teórico-prático sobre aspectos legais e advocacia em saúde, na área de pediatria comunitária, em programas de residência médico-pediátrica, demonstraram um reconhecimento da possibilidade de se necessitar de articulação entre as questões legais e de saúde ao se assistir um paciente $\mathrm{e}^{(13,16)}$.

\section{DISCUSSÃO}

Essa revisão possibilitou identificar uma valorização da comunidade como contexto de intervenções em saúde, na qual está presente uma ênfase no ensino de competências e habilidades de advocacia em saúde da criança. O exercício de advocacia em saúde da criança pode ser explorado na atuação de profissionais de saúde, como enfermeiros e médicos, entre outros.

Parcerias e identificação de recursos na comunidade receberam ênfase. Considerando que uma atitude de defesa pela saúde da criança engloba múltiplos fatores, numa compreensão ampla de processo saúde-doença e num momento em que determinantes sociais têm se destacado nesse processo, a atuação isolada do profissional e do setor saúde se torna muitas vezes incapaz de alcançar a resolutividade almejada para cada caso, tornando um imperativo que o profissional de saúde, exercendo conscientemente o papel de advogado pela saúde daqueles que assiste, desenvolva um processo de comunicação para estabelecimento de parcerias com outros setores, bem como com recursos da própria comunidade, valorizando serviços governamentais e não governamentais.

Na assistência à infância, essas parcerias são potenciais, tendo em vista a amplitude e destaque das organizações sociais dirigidas às crianças, tornando importante o debate sobre políticas públicas que visem a estabelecer e a fortalecer esses vínculos no âmbito local e nacional(20).

Diante de uma vulnerabilidade ou privação vivenciada pela criança, o profissional de saúde deve identificar alternativas potenciais de resolução, colocando o foco nas possibilidades e não nas dificuldades, devendo buscar uma diversidade de recursos na comunidade e competências profissionais para exercer esse papel de mediador e facilitador, que caracteriza o papel de advogado.

Assim, atenta-se para a possibilidade de parcerias intersetoriais e interinstitucionais, com conseqüente construção conjunta de iniciativas para a solução de problemas públicos, evitando a negação ao Estado e a fragmentação das ações ${ }^{(21)}$ Organizações externas à instituição governamental têm também possibilidades de contribuir para a consolidação de técnicas de trabalho que refletem um enfoque integrado e que incluem métodos de participação dirigidos a fortalecer o processo de reforma no setor saúde, o que é favorecido pelo seu permanente contato com a sociedade civil e seus diversos atores sociais ${ }^{(22)}$.

Nesse sentido, consideramos que o enfermeiro é um profissional que desempenha importantes papéis para o cuidado, na educação em saúde, nas políticas públicas, na interlocução entre vários atores sociais, tendo um contato particularmente próximo dos usuários, profissionais, gestores, conselhos, lideranças comunitárias, coordenadores de serviços e setores.

No exercício da advocacia em saúde, manifesta-se a necessidade de estabelecimento de uma relação intersetorial entre a área da saúde e a área de direito, numa demanda por serviços de referência e também por recursos e instrumentos para o profissional de saúde atuar sob uma ótica mais ampla.

Na área de saúde mental já são relatadas algumas iniciativas de articulação entre serviços prestados por profissionais da saúde e do direito, como um estudo que descreve a experiência de um Escritório de Advocacia para Direitos, Saúde Mental \& Cidadania, que funciona como um dispositivo de agenciamento social, que promove parcerias e alianças entre as instituições prestadoras de assistência à Saúde e os movimentos organizados como a Ordem dos Advogados do Brasil, a Comissão dos Direitos Humanos e o Ministério Público ${ }^{(23)}$. Consideramos que a institucionalização de parcerias como essa, direcionadas à clientela infantil, são viáveis e podem potencializar a utilização de instrumentos legais, como o Estatuto da Criança e do Adolescente, e de serviços, como o Conselho Tutelar, incluindo ativamente os pais e professores, que se constituem elementos importantes na vida das crianças, o que foi reconhecido nos artigos analisados.

Um estudo que revisou as leis, resoluções e outros documentos referentes ao direito das crianças e adolescentes existentes no Brasil e exterior destacaram como documentos importantes a Declaração dos Direitos da Criança de 1959; a Constituição Federal do Brasil de 1988; a Carta da Criança Hospitalizada de 1988; o Estatuto da Criança e do Adolescente de 1990; e os Direitos da Criança e do Adolescente Hospitalizados (Resolução 41/95), concluindo que já existem legislações que protegem o direito da criança, mas que, porém, é necessário garantir o usufruto desses direitos ${ }^{(24)}$.

A estimulação da participação de atores sociais diversos na defesa pela saúde das crianças, por parte dos profissionais de saúde, busca constituir-se como uma prática de emancipação, embora em relação aos limites no impacto produzido pelas ações de advocacia em saúde da criança, os resultados de um dos estudos desta revisão demonstraram que na experiência relatada, mudanças de comportamento continuam sendo um desafio(11)

Internacionalmente, o conceito de advocacia foi definido como ação positiva que resulta em mudança e a defesa como uma parte fundamental na interação enfermeiro-paciente, sendo que os enfermeiros representam fortes advogados dos pacientes ${ }^{(25)}$. Para o exercício da advocacia em saúde é necessário tomar para si a iniciativa de direcionar esforços, o que caracteriza o papel do advogado. Partindo dessa premissa, considera-se que qualquer indivíduo é um potencial 
advogado e o enfermeiro possui particular perfil profissional para desenvolver essa atuação.

\section{CONSIDERAÇÕES FINAIS}

Há um reconhecimento atual sobre a vulnerabilidade na infância e sobre o destaque dos determinantes sociais no processo saúde-doença, levando a um contexto em que intervenções clínicas isoladas ou restritas ao setor saúde são cada vez mais consideradas inócuas. O profissional de saúde é um elemento especialmente munido de condições para identificar e compreender melhor a situação particular de vulnerabilidade apresentada por uma criança e seu entorno familiar e comunitário. E diante, então, do cenário reconhecido, muitas vezes torna-se necessário e desejável o exercício de atividades e habilidades de advocacia em prol da criança, numa atuação que vai além da intervenção clínica e tende a extrapolar os limites do setor saúde.
Essa atuação envolve uma postura pró-ativa do profissional, na busca de parcerias familiares, comunitárias, interdisciplinares e intersetoriais. Considera-se que profissionais no contexto da atenção primária de saúde têm possibilidades favoráveis para desenvolver essa atuação, condizente especialmente com as propostas e princípios norteadores da Estratégia Saúde da Família (ESF), em nosso país.

Tendo em vista a ESF, destacamos, no presente trabalho, uma reflexão sobre o potencial do enfermeiro como advogado em saúde da criança e sua família, sendo esse um profissional-chave dentro da equipe de saúde da família e possuindo uma formação acadêmica ampla, que permite o desenvolvimento de habilidades diversas, importantes no exercício da advocacia em saúde.

Nesse sentido, seria de especial contribuição, pesquisas que se ocupassem de estudar a significância desse conceito em nossa realidade, abordando as possibilidades de sua aplicabilidade no cotidiano dos serviços de saúde, tendo em vista aspectos facilitadores e limitadores.

\section{REFERÊNCIAS}

1. Shiratori K, Costa TL, Formozo GA, Silva SA. Educação em saúde como estratégia para garantir a dignidade da pessoa humana. Rev Bras Enferm [periódico na Internet]. 2004 Out [citado 2010 Maio 17];57(5):617-619. Disponível em: http://www.scielo.br/scielo.php?script = sci arttext\&pid $=$ S0034-71672004000500021\&Ing = pt.doi: 10.1590/S0034-71672004000500021.

2. Dallari SG, Adorno RCF, Faria MM, Shuqair NMSAQ, Trewikowski S. O direito à saúde na visão de um conselho municipal de saúde. Cad. Saúde Pública [periódico na Internet]. 1996 Dec [citado 2010 Maio 17];12(4):531-540. Disponível em: http://www.scielo.br/scielo.php?script =sci arttext\&pid = S0102-311X1996000400012\&lng = en.doi: 10.1590/S0102-311X1996000400012.

3. Blank D. Controle de injúrias sob a ótica da pediatria contextual. J. Pediatr. (Rio J.) [periódico na Internet]. 2005 Nov [citado 2010 Maio 17];81(5), Disponível em: http:// www.scielo.br/scielo.php?script $=$ sci_arttext\&pid $=$ S0021-75572005000700002\&Ing=en.doi:10.1590/ S0021-75572005000700002

4. Dallari SG, Barber-Madden R, Torres-Fernandes MC, Shuqair NSMSAQ, Watanabe HA. Advocacia em saúde no Brasil contemporâneo. Rev. Saúde Públ. [periódico na Internet]. 1996 Dez [citado 2010 Maio 17];30(6):592-601. Disponível em: http://www.scielosp.org/scielo.php?script =sci arttext\&pid = S0034-89101996000600014\&Ing = en.doi: 10.1590/S0034-89101996000600014.

5. Figueiredo GLA, Mello DF. Atenção à saúde da criança no Brasil: aspectos da vulnerabilidade programática e dos direitos humanos. Rev. Latino-Am. Enfermagem [periódico na Internet]. 2007 Dez [citado 2010 Maio 17];15(6):1171-1176. Disponível em: http://www.scielo.br/scielo.php?script = sci arttext\&pid = S0104-11692007000600018\&Ing = en.doi: 10.1590/S0104-11692007000600018

6. Cordeiro AM et al. Revisão sistemática: uma revisão narrativa. Comunicação Científica 2007;34(6):428-431.
7. Hufford L, West DC, Paterniti DA, Pan RJ. Communitybased advocacy training: applying asset-based community development in resident education. Acad Med 2009;84(6):765-70.

8. Waterston T. Teaching and learning about advocacy. Arch Dis Child Educ Pract Ed 2009;94(1):24-8.

9. Au H, Harrison M, Ahmet A, Orsino A, Beck CE, Tallet $S$ et al. Residents as health advocates: the development, implementation and evaluation of a child advocacy initiatives at the University of Toronto (Toronto, Ontario). Paediatr Child Health 2007;12(7):567-72.

10. Keller D, Jones N, Savageau JA, Cashman SB. Development of a brief questionnaire to identify families in needs of legal advocacy to improve child health. Ambul Pediatr 2008;8(4):266-9.

11. Grant N, Gibbs T, Naseeb TA, Al-Garf A. Medical students as family-health advocates: Arabian Gulf University experience. Med Teach 2007;29(5):117-21.

12. Raman S, Woolfenden S, Williams K, Zwi K. Human rights and child health. J Paediatr Child Health 2007;43(9):581-6.

13. Solomon BS, Minkovitz CS, Grason HA, Carraccio C. Community pediatrics: a consistent focus in residency training from 2002 to 2005. Ambul Pediatr 2007;7(4):321-4.

14. Cohen BE, Reutter L. Development of the role of public health nurses in addressing chid and family poverty: a framework for action. J Adv Nurs 2007;60(1):96-107.

15. Mclntosh J, Shute J. The process of health visiting and its contribution to parental support in the Starting Well demonstration project. Health Soc Care Community 2007;15(1):77-85.

16. MinKovitz CS, Chandra A, Solomon BS, McDonnell KA, Silver GB, Tonniges TF. Communit y Pediatrics: gender differences in perspectives of residents. Ambul Pediatr 2006;6(6):326-31. 
17. Paterniti DA, Pan RJ, Smith LF, Horan NM, West DC. From physician-centered to community-oriented perspectives on health care: assessing the efficacy of communitybased training. Acad Med 2006;81(4):347-53.

18. Chamberlain LJ, Sanders LM, Takayama JI. Child advocacy training: curriculum outcomes and resident satisfaction. Arch Pediatr Adolesc Med 2005;159(9):842-7.

19. Shipley LJ, Stelzner SM, Zenni EA, Hargunani D, O' Keefe $\mathrm{J}$, Miller $\mathrm{C}$ et al. Teaching community pediatrics to pediatric residents: strategic approaches and successful models for education in community health and child advocacy. Pediatrics 2005;115(4):1150-7.

20. Andrade RD, Mello DF. Organizações sociais e instituições governamentais: perspectivas de parceria na atenção à saúde da criança através dos voluntários e da pastoral da criança. Rev Esc Enferm USP 2006;40(1):93-7.

21. Magalhães R. Enfrentando a pobreza, reconstruindo vínculos sociais: as lições da Ação da Cidadania contra a Fome, a Miséria e pela Vida. Cad Saúde Pública. 2002;18(supl):121-37.
22. Dickson K. Colaboracion entre organizaciones no gubernamentales y los gobiernos en la reforma del sector de la salud. Rev Panam Salud-Publica. 1997;1(4):324-9.

23. Mota TD, Barros S. Saúde mental, direitos, cidadania: o escritório de advocacia como agência para inclusão social. Rev Esc Enferm USP [periódico na Internet]. 2008 Junho [citado 2010 Maio 17];42(2):220-226. Disponível em: http://www.scielo.br/scielo.php?script $=$ sci arttext\&pid $=$ S0080-62342008000200002\&lng $=$ en. doi: 10.1590/S0080-62342008000200002.

24. Gomes ILV, Caetano R, Jorge MSB. A criança e seus direitos na família e na sociedade: uma cartografia das leis e resoluções. Rev Bras Enferm [periódico na Internet]. 2008 Fev [citado 2010 Maio 17];61(1):61-65. Disponível em: http://www.scielo.br/scielo.php?script = sci arttext\&pid $=$ S0034-71672008000100009\&Ing = pt.doi: 10.1590/S0034-71672008000100009.

25. International Training and Educational Center on HIV (I$\mathrm{TECH}$ ). Building global capacity to improve the care of people living with HIV/AID. Disponível em:http://www.go2itech.org/ppt/p06-db/db-51029-60.ppt. Acesso em: jul. 2008. 